\title{
A Quartic System with Twenty-Six Limit Cycles
}

\author{
Tomas Johnson
}

CONTENTS

1. Introduction

2. The Construction

3. Numerical Issues

4. Numerical Results

References

2000 AMS Subject Classification: Primary: 34C07; Secondary: 37G15, 37G40, 37M20, 65G20

Keywords: Pseudo-Abelian integrals, limit cycles, bifurcation theory, planar integrable systems, interval analysis
We construct a planar quartic system and demonstrate that it has at least 26 limit cycles. The vector field is symmetric and integrable, but non-Hamiltonian. The proof is based on a verified computation of zeros of pseudo-Abelian integrals, together with the symmetry properties.

\section{INTRODUCTION}

Bifurcations of limit cycles from planar polynomial vector fields is connected to the second part of Hilbert's sixteenth problem, which asks for an upper bound, $H(n)$, on the number of limit cycles that an $n$ th-degree planar polynomial vector field can have. The determination of $H(n)$ has turned out to be very difficult. In fact, it is not even known whether $H(n)$ exists. A thorough review of this problem and the progress that has been made toward its solution can be found in [Il'yashenko 02, Roussarie 98].

There are various restricted versions of the problem, the most thoroughly studied being probably the weak Hilbert's sixteenth problem, formulated by Arnol'd [Arnol'd 90, Christopher and Li 07]. The weak problem asks for an upper bound, $Z(n)$, on the number of limit cycles that can bifurcate from a planar Hamiltonian vector field under first-order perturbation. Obviously, $Z(n) \leq H(n)$. The weak problem has been solved for $n=2, Z(2)=2$ [Chen et al. 06]. For $n>2$, it is known that $Z(n)$ exists (see, e.g., [Varchenko 84]), but there are no realistic upper bounds on its growth.

Based on the difficulties in obtaining upper bounds on the number of limit cycles of a planar polynomial vector field, there has been a large interest in the computation of lower bounds of $H(n)$ and $Z(n)$. Some such bounds are $Z(3) \geq 13$ [Li et al. 09], $Z(4) \geq 16$ [Wang et al. 08], $Z(5) \geq 27$ [Johnson and Tucker 10a], $Z(7) \geq 53$ [Johnson and Tucker 10b], and in addition $H(4) \geq 22$ [Christopher and Li 07]. The results for the weak problem for odd degrees are proved by calculating the numbers of zeros of Abelian integrals, whereas 
the results for quartic vector fields are proved by computing focus values at a linear center. In this paper we improve the result for quartic vector fields and prove that $H(4) \geq 26$.

The construction in this paper is based on firstorder perturbation of an integrable non-Hamiltonian planar vector field with maximum number of centers and $\mathbb{Z}_{2}$ symmetry. The method, however, is general, and could be applied to the study of perturbations around any center where one is able to parameterize the level curves. A detailed analysis of the impact of symmetry on cubic and quartic vector fields is done in [Yu et al. 06]. Most studies on the bifurcation of limit cycles under first-order perturbations are done for perturbations of Hamiltonian vector fields; some other papers considering integrable non-Hamiltonian systems are [Han et al. 07, Yu and Li 02]. Our proof is done using verified numerical computations based on interval analysis [Moore 66, Neumaier 90]. The details of the numerical computations, which are very delicate, are described in Section 3. Other computer-aided approaches to the determination of phase portraits of planar vector fields include [Guckenheimer 95, Guckenheimer and Malo 96, Johnson 09, Johnson and Tucker 09, Johnson and Tucker 11]. A computational algebra approach to the center problem can be found in [Romanovski and Shafer 09].

The outline of this paper is as follows: In Section 2, we describe our construction and the theoretical background to our results. Some information about computer-aided proofs and the numerical issues involved is given in Section 3. Finally, in Section 4, we describe the results of the computations.

We end this introduction by stating our main theorem.

Theorem 1.1. There exist a quartic integrable planar vector field and a quartic perturbation such that the perturbed system has at least 26 limit cycles when the perturbation is sufficiently small.

\section{THE CONSTRUCTION}

As in [Johnson and Tucker 10a, Johnson and Tucker 10b], we study vector fields with maximal number of centers and $\mathbb{Z}_{2}$ symmetric first integrals. We study bifurcations from a quartic system whose first integral is equal to the Hamiltonian of a cubic Hamiltonian system with maximal number of centers:

$$
\dot{x}=-y^{2}\left(y^{2}-1.1\right), \quad \dot{y}=x y\left(x^{2}-0.9\right) \text {. }
$$

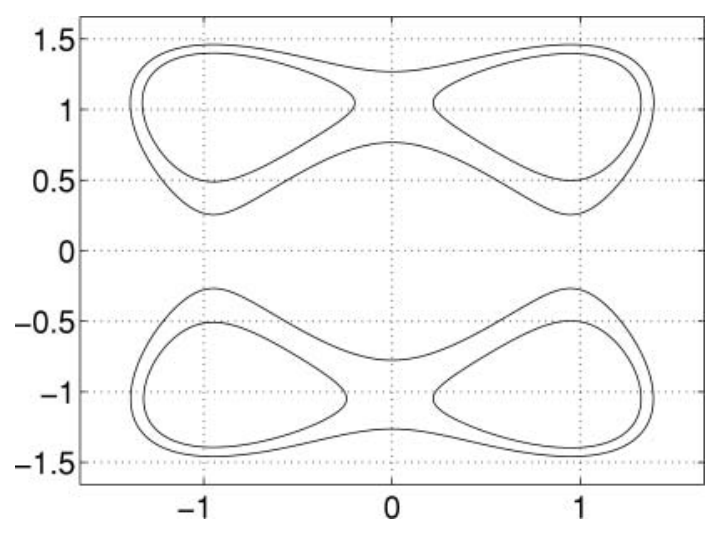

FIGURE 1. The phase portrait of system $(2-1)$.

Clearly, an integrating factor of $(2-1)$ is $\mu=1 / y$, and the corresponding first integral is

$$
F(x, y)=\frac{1}{4}\left(x^{4}+y^{4}\right)-\frac{1}{20}\left(9 x^{2}+11 y^{2}\right),
$$

which is $\mathbb{Z}_{2}$ symmetric. The system (2-1) has six annuli of periodic orbits, appearing in two classes: $\Gamma_{1}$ (multiplicity 4) and $\Gamma_{2}$ (multiplicity 2); see Figure 1.

To use the symmetry of the system, we consider perturbations with the same kind of symmetry. Through the use of perturbations that respect the symmetry, any limit cycle that we detect will imply the existence of three additional ones if it belongs to the first class of periodic annuli, and one additional limit cycle if it belongs to the second class. The perturbed system that we consider is the following:

$$
\begin{aligned}
\dot{x}= & -y^{2}\left(y^{2}-1.1\right) \\
\dot{y}= & x y\left(x^{2}-0.9\right) \\
& +\epsilon\left(x^{2} y^{2}+\frac{\alpha_{1}}{3} y^{4}-\alpha_{2}-\alpha_{3} x^{2}-\alpha_{4} x^{4}\right),
\end{aligned}
$$

where $\epsilon>0$ is a small parameter. We denote the perturbation by $\epsilon g(x, y)$.

Each annulus of periodic orbits corresponds to a continuous family of level curves of the first integral $\gamma_{h} \subset F^{-1}(h)$. The classical method of studying perturbations from such an annulus in the Hamiltonian case is to study Abelian integrals; see, e.g., [Christopher and Li 07, Guckenheimer and Holmes 83]. In the present, non-Hamiltonian setting, the corresponding objects are pseudo-Abelian integrals, which have similar properties. Given a perturbation as in $(2-3)$, we define the pseudo-Abelian integral (in general multivalued) as

$$
I(h)=\int_{\Gamma_{h}}-\mu(x, y) g(x, y) d x
$$




\begin{tabular}{lr}
\hline$\alpha_{1}$ & -44.1527847886279 \\
$\alpha_{2}$ & -64.7283580682510 \\
$\alpha_{3}$ & 155.6409265346564 \\
$\alpha_{4}$ & -28.2884027418255 \\
\hline
\end{tabular}

TABLE 1. The generated coefficients of the perturbation $(2-3)$.

which in our case reads

$I(h)=\int_{\Gamma_{h}}\left(-x^{2} y-\frac{\alpha_{1}}{3} y^{3}+\alpha_{2} \frac{1}{y}+\alpha_{3} \frac{x^{2}}{y}+\alpha_{4} \frac{x^{4}}{y}\right) d x$.

The most important property of (pseudo-)Abelian integrals is described by the Poincaré-Pontryagin theorem.

Theorem 2.1. (Poincaré-Pontryagin.) Let $P$ be the return map defined on some section transversal to the level curves of $F$, parameterized by the values $h$ of $F$, where $h$ is taken from some bounded interval $(a, b)$. Let $d(h)=P(h)-h$ be the displacement function. Then $d(h)=\epsilon(I(h)+\epsilon \phi(h, \epsilon))$, as $\epsilon \rightarrow 0$, where $\phi(h, \epsilon)$ is analytic and uniformly bounded on a compact neighborhood of $\epsilon=0, h \in(a, b)$.

For a proof of this theorem, see, for example, [Christopher and Li 07].

As a consequence of the above theorem, one can prove that a simple zero of $I(h)$ corresponds to a unique limit cycle bifurcating from the integrable system as $\epsilon \rightarrow 0$. In fact, to prove the existence of a limit cycle, it suffices to have a zero of odd order.

Clearly, if (2-4) is zero on one level curve in one of the annuli of periodic orbits in a class, it is zero on the other ones, since the different annuli are reflections of each other in the $x$ - or $y$-axis. This yields the claimed multiplicity of limit cycles.

\section{NUMERICAL ISSUES}

The proof of Theorem 1.1 is computer-aided. In order to use a computer to prove mathematical statements, the results of a computation must be guaranteed to be correct. We need to prove that a given mathematical statement can be reduced to a finite number of computable conditions, and construct an algorithm that checks those conditions.

A numerical algorithm is said to be auto-validating if it guaranteed to produce a mathematically correct re-
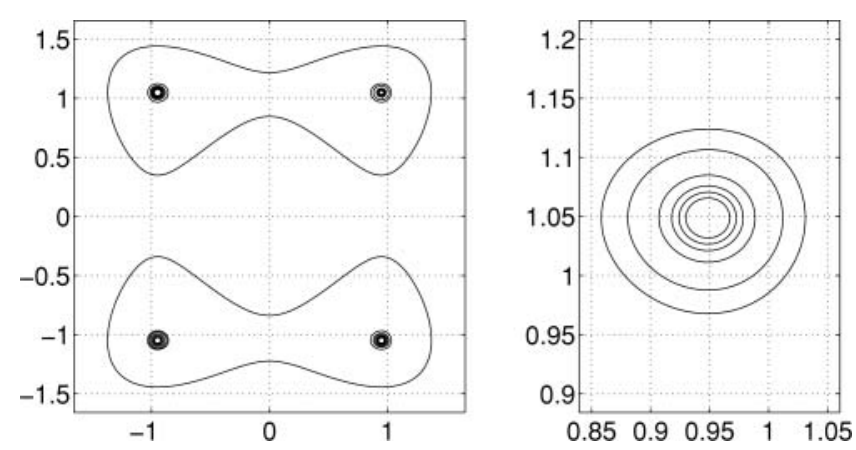

FIGURE 2. The level curves from which the limit cycles bifurcate. On the right is a closeup of $\Gamma_{1}$.

sult. The basic object in any such algorithm is an interval whose endpoints are computer-representable floatingpoint numbers. Replacing numbers with intervals yields an arithmetic for sets. Computing with sets, rather than points, we can quantify all discretization errors of a numerical algorithm. Since any bounded subset of the plane can be covered by a finite number of axis-parallel boxes, we can, for example, do computations that are valid for the entire domain of a function, or all functions in a finitely parameterized family. In addition to the discretization errors of a numerical method, an auto-validated numerical algorithm also incorporates the computer's internal representation of the floating-point numbers and its rounding procedures. All mathematical operations are performed in interval arithmetic with directed rounding to ensure the correctness of the result; see, e.g., [Moore 66, Neumaier 90] for details. Our

\begin{tabular}{lll} 
P.A. & \multicolumn{1}{l}{$h$} & $I(h)$ \\
\hline 1 & -0.5045 & {$[-9.616 \mathrm{E}-0013,-9.542 \mathrm{E}-0013]$} \\
1 & -0.5035 & {$[4.982 \mathrm{E}-0013,5.027 \mathrm{E}-0013]$} \\
1 & -0.5025 & {$[-4.766 \mathrm{E}-0013,-4.690 \mathrm{E}-0013]$} \\
1 & -0.5018 & {$[1.730 \mathrm{E}-0012,1.742 \mathrm{E}-0012]$} \\
1 & -0.5010 & {$[-6.946 \mathrm{E}-0012,-6.931 \mathrm{E}-0012]$} \\
1 & -0.5000 & {$[1.052 \mathrm{E}-0011,1.055 \mathrm{E}-0011]$} \\
1 & -0.4500 & {$[-9.858 \mathrm{E}-0005,-9.857 \mathrm{E}-0005]$} \\
2 & -0.2900 & {$[-6.018 \mathrm{E}+0000,-6.017 \mathrm{E}+0000]$} \\
2 & -0.2500 & {$[1.145 \mathrm{E}+0001,1.146 \mathrm{E}+0001]$}
\end{tabular}

TABLE 2. The computed enclosures of the pseudoAbelian integrals. P.A. = periodic annulus. 

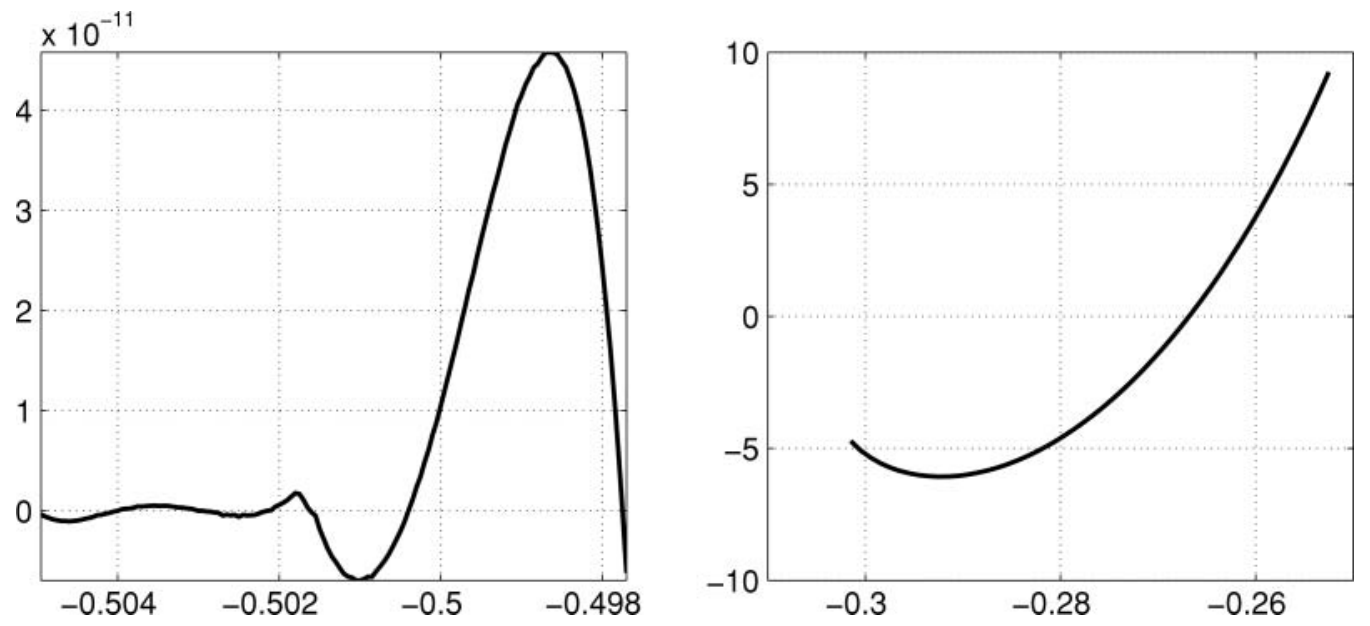

FIGURE 3. The graphs of $I(h)$ on $\Gamma_{1}$ and $\Gamma_{2}$.

algorithms use the interval arithmetic package C-XSC [Hammer et al. 95]. ${ }^{1}$

In the case at hand, we need to rigorously enclose the value of $I(h)$ in $(2-4)$. We do this using the interval extension of Simpson's method, i.e.,

$$
\begin{aligned}
\int_{a}^{b} f(x) d x \in & \frac{b-a}{6}\left(f(a)+4 f\left(\frac{a+b}{2}\right)+f(b)\right) \\
& -\frac{1}{2880}(b-a)^{5} f^{(4)}([a, b]) .
\end{aligned}
$$

Note that $f^{(4)}([a, b])$ in the above formula denotes an interval enclosure of the range of $f^{(4)}$ on the interval $[a, b]$. In order to enclose the error term in Simpson's method, we need differentiable information about the function. To get the enclosures of the required derivatives, we compute with Taylor arithmetic (the necessary software is provided by [Blomquist et al. 05]), which uses automatic differentiation (see, e.g., [Griewank 00]), to compute the derivatives of a function.

The simplicity of the first integral (2-2) implies that we can determine, by hand, the parts of a given level curve $\gamma_{h}$ where $y=y(x)$ and $x=x(y)$. This reduces the computation of $I(h)$ to one-dimensional integration. With this parameterization, however, the function we are integrating contains a factor of the form $\sqrt{\cdots+\sqrt{\cdots}}$, which is numerically unstable. When we compute higherorder derivatives, even more accuracy is lost. The choice of Simpson's method rather than a higher-order Taylor integration scheme is a compromise between order and accuracy of enclosures. The size of the enclosures of

${ }^{1} \mathrm{CXSC}, \mathrm{C}++$ eXtension for Scientific Computation, version 2.2.3. Available from http://www.math.uni-wuppertal.de/org/WRST/ xsc/cxsc.html. higher-order derivatives computed with automatic differentiation will grow very fast with the order.

In addition, the value of a pseudo-Abelian integral with many zeros is very small; in our case, the sum in $(2-4)$ is up to 14 orders of magnitude smaller than each of its terms. This huge demand for accuracy together with the inaccuracy of implicitly defined functions and the fact that the square root is non-Lipschitz at zero, which implies that the relative size of the interval enclosures grows without bound close to zero, makes the computations extremely delicate. In fact, it forces us to compute with 256-bit arithmetic. With lower-precision computations we get only enclosures of the values of $(2-4)$ of the form $(-\eta, \eta)$, for some small number $\eta$. This illustrates the strength of validated numerical methods; we are able to tell when we have enough accuracy. Using a standard numerical experiment for a problem this sensitive, any result is possible.

\section{NUMERICAL RESULTS}

The idea is to generate a perturbation with as many zeros as possible in $\Gamma_{1}$, and afterward check whether zeros in $\Gamma_{2}$ are also implied. In contrast to an algebraic approach, such as [Romanovski and Shafer 09], we do not compute focus quantities. Instead, we compute the zeros of the pseudo-Abelian integral, for some fixed perturbation. This means that the limit cycles will have some distance from the center.

In trying to locate a perturbation with as many zeros as possible, we start by (nonrigorously) sampling the value of each of the five terms in (2-4) at 100 uniformly distributed level curves in $-0.505 \leq h \leq-0.5$, 
where -0.505 corresponds to the center. Since we have four coefficients in our perturbation, we can solve the linear system constructed by requiring that $I(h)=0$ at four different $h$-values. We do this with the four values as close as possible to the center, and evenly spaced. After some small corrections, we get the choice of parameters given in Table 1, yielding six limit cycles in $\Gamma_{1}$ and one limit cycle in $\Gamma_{2}$. These are our candidate coefficients, and the final step is to run the validated integration scheme to prove that $I(h)$ has validated sign changes.

The level curves from which the limit cycles bifurcate are shown in Figure 2, the graphs of the pseudo-Abelian integrals on $\Gamma_{1}$ and $\Gamma_{2}$ are presented in Figure 3, and the validated enclosures of the values are given in Table 2 .

\section{REFERENCES}

[Arnol'd 90] V. I. Arnol'd. "Ten Problems." In Theory of Singularities and Its Applications, Adv. Soviet Math., 1, pp. 1-8. Providence: Amer. Math. Soc, 1990.

[Blomquist et al. 05] F. Blomquist, W. Hofschuster, and W. Krämer. "Real and Complex Taylor Arithmetic in CXSC." Preprint 2005/4, Universität Wuppertal. Available online (http://www.math.uni-wuppertal.de/ $\mathrm{xsc}$ ), 2005 .

[Chen et al. 06] F. Chen, C. Li, J. Llibre, and Z. Zhang. "A Unified Proof on the Weak Hilbert 16th Problem for $n=$ 2." J. Differential Equations 221:2 (2006), 309-342.

[Christopher 05] C. Christopher. "Estimating Limit Cycle Bifurcations from Centers." In Differential Equations with Symbolic Computation, Trends Math., pp. 23-35. Basel: Birkhäuser, 2005.

[Christopher and Li 07] C. Christopher and C. Li. Limit Cycles of Differential Equations, Advanced Courses in Mathematics, CRM Barcelona. Basel: Birkhäuser, 2007.

[Griewank 00] A. Griewank. Evaluating Derivatives: Principles and Techniques of Algorithmic Differentiation, SIAM Frontiers in Applied Mathematics. Philadelphia: SIAM, 2000 .

[Guckenheimer 95] J. Guckenheimer. "Phase Portraits of Planar Vector Fields: Computer Proofs." Experiment. Math. 4:2 (1995), 153-165.

[Guckenheimer and Holmes 83] J. Guckenheimer and P. Holmes. Nonlinear Oscillations, Dynamical Systems, and Bifurcations of Vector Fields, Applied Mathematical Sciences 42. New York: Springer-Verlag, 1983.
[Guckenheimer and Malo 96] J. Guckenheimer and S. Malo. "Computer-Generated Proofs of Phase Portraits for Planar Systems." Internat. J. Bifur. Chaos Appl. Sci. Engrg. 6:5 (1996), 889-892.

[Hammer et al. 95] R. Hammer, M. Hocks, U. Kulisch, and D. Ratz. $C++$ Toolbox for Verified Computing. New York: Springer-Verlag, 1995.

[Han et al. 07] M. Han, D. Shang, W. Zheng, and P. Yu. "Bifurcation of Limit Cycles in a Fourth-Order NearHamiltonian System." Internat. J. Bifur. Chaos Appl. Sci. Engrg. 17:11 (2007), 4117-4144.

[Il'yashenko 02] Yu. S. Il'yashenko. "Centennial History of Hilbert's 16th Problem." Bull. Amer. Math. Soc. (N.S.) 39:3 (2002), 301-354.

[Johnson 09] T. Johnson. "Computer-Aided Computation of Abelian Integrals and Robust Normal Forms." PhD thesis, Uppsala University, 2009.

[Johnson and Tucker 09] T. Johnson and W. Tucker. "A Rigorous Study of Possible Configurations of Limit Cycles Bifurcating from a Hyper-elliptic Hamiltonian of Degree Five." Dynamical Systems: An International Journal $24: 2$ (2009), 237-247.

[Johnson and Tucker 10a] T. Johnson and W. Tucker. "An Improved Lower Bound on the Number of Limit Cycles Bifurcating from a Quintic Hamiltonian Planar Vector Field under Quintic Perturbation." International Journal of Bifurcation and Chaos in Applied Sciences and Engineering, 20:1 (2010), 63-70.

[Johnson and Tucker 10b] T. Johnson and W. Tucker. "An Improved Lower Bound on the Number of Limit Cycles Bifurcating from a Hamiltonian Planar Vector Field of Degree 7." International Journal of Bifurcation and Chaos in Applied Sciences and Engineering, 20:5 (2010), 1451-1458.

[Johnson and Tucker 11] T. Johnson and W. Tucker. "On a Computer-Aided Approach to the Computation of Abelian Integrals." To appear in BIT-Numerical Mathematics, 2011.

[Li et al. 09] C. Li, C. Liu, and J. Yang. "A Cubic System with Thirteen Limit Cycles." J. Differential Equations 246:9 (2009), 3609-3619.

[Moore 66] R. E. Moore. Interval Analysis. Englewood Cliffs, NJ: Prentice-Hall, 1966.

[Neumaier 90] A. Neumaier, "Interval Methods for Systems of Equations." In Encyclopedia of Mathematics and Its Applications 37. Cambridge, UK: Cambridge Univ. Press, 1990. 
[Romanovski and Shafer 09] V. G. Romanovski and D. S. Shafer. The Center and Cyclicity Problems: A Computational Algebra Approach. Boston: Birkhäuser, 2009 .

[Roussarie 98] R. Roussarie. Bifurcation of Planar Vector Fields and Hilbert's Sixteenth Problem, Progress in Mathematics 164. Basel: Birkhäuser, 1998.

[Varchenko 84] A. N. Varchenko. "Estimation of the Number of Zeros of an Abelian Integral Depending on a Parameter, and Limit Cycles." Funktsional. Anal. i Prilozhen. 18:2 (1984), 14-25.
[Wang et al. 08] Q. Wang, Y. Liu, and C. Du. "Small Limit Cycles Bifurcating from Fine Focus Points in Quartic Order $Z_{3}$-Equivariant Vector Fields." J. Math. Anal. Appl. $337: 1$ (2008), 524-536.

[Yu and Li 02] J. Yu and C. Li. "Bifurcation of a Class of Planar Non-Hamiltonian Integrable Systems with One Center and One-Homoclinic Loop." J. Math. Anal. Appl. 269:1 (2002), 227-243.

[Yu et al. 06] P. Yu, M. Han, and Y. Yuan. "Analysis on Limit Cycles of $Z_{q}$-Equivariant Polynomial Vector Fields with Degree 3 or 4" (English summary). J. Math. Anal. Appl. 322:1 (2006), 51-65.

Tomas Johnson, Institut Mittag-Leffler, Auravägen 17, SE-182 60 Djursholm, Sweden (tomas.johnson@math.uu.se)

Received December 18, 2009; accepted March 23, 2010. 